\title{
Genotypic diagnosis of familial Mediterranean fever (FMF) using new microsatellite markers: example of two extensive non-Ashkenazi Jewish pedigrees
}

CNRS URA 1922, Généthon, Evry, France

C Clépet

Unité de Recherche en Epidémiologie et

Immuno-oncologie

Pédiatriques, Faculté de Médecine Nord,

13326 Marseille Cedex

15, France

I Koné-Paut

Correspondence to: Dr Touitou.

Received 19 July 1996 Revised version accepted for publication 3 January 1997

\begin{abstract}
Laboratoire de
Génétique Moléculaire et Chromosomique, Hôpital A de

Villeneuve, $371 \mathrm{Av}$ du Doyen Gaston Giraud, 34295 Montpellier

Cedex 5, France

M Dupont

C Dross

I Gourdier

J Demaille

I Touitou

EA1501, Biologie

Moléculaire des

Cellules Eucaryotes,

ICGM, Université

Paris 5, Paris, France

N Smaoui

B Nedelec

G Grateau

M Delpech

Abstract

Familial Mediterranean fever is an autosomal recessive disease characterised by multiple attacks of serosal inflammation in the absence of treatment. In the absence of timely diagnosis, renal amyloidosis is a life threatening complication. The diagnosis is often missed because no specific test is available. Early colchicine treatment prevents attacks and renal complications. The FMF gene (MEF) has been mapped to chromosome 16 p13.3 but has not yet been identified. We compared the suitability of a series of microsatellite markers (four of them were new) and propose the routine use of seven of these markers, exhibiting alleles in strong linkage disequilibrium with the disease and informative in $100 \%$ of diagnosed patients. Moreover, the discovery of a homozygous status for the 3-3-9 (or 3-3-18) haplotype at the core loci $(\mathrm{D} 16 \mathrm{S3070}, \mathrm{D16S3082}$, and D16S3275), which was found in $73 \%$ non-Ashkenazi Jewish patients, points to a diagnosis of FMF, even in sporadic cases, with a risk of error of only $2.10^{-5}$. Two extensive pedigrees covering most indications for genetic counselling are presented, showing that it is now possible both prospectively and retrospectively to identify members likely to have MEF mutations. With the help of this accurate test, colchicine treatment can be better targeted, especially where the symptomatology is mild or atypical.

(F Med Genet 1997;34:375-381)
\end{abstract}

Madeleine Dupont, Christiane Dross, Nizar Smaoui, Brigitte Nedelec, Gilles Grateau, Christian Clépet, Isabelle Gourdier, Isabelle Koné-Paut, Marc Delpech, Jacques Demaille, Isabelle Touitou

Keywords: FMF; genotypic diagnosis; chromosome 16, microsatellite markers

Familial Mediterranean fever is one of the most frequent hereditary disorders in nonAshkenazi Jews. ${ }^{1}$ A recent study showed a very high FMF gene frequency in all ethnic groups originating from North African countries. ${ }^{2}$ In Israel, heterozygote frequencies range from 1:5 to $1: 30$, with a mean heterozygosity rate of $\sim$ 1:8. FMF also affects other populations of Mediterranean ancestry (Armenians, Turks, and Arabs). Since no specific biological defect is known, diagnosis so far has been solely based on clinical, ethnic, or familial features.
FMF is characterised by recurrent short attacks of fever associated with acute abdominal (95\%), joint (75\%), and chest (50\%) pain. ${ }^{3}$ Fever and pain are caused by inflammation of the corresponding serosal membranes (peritoneum, synovia, or pleura). Most patients experience the disease before the age of 30 . In some cases, clinical signs are not typical (isolated orchitis or meningitis) or symptoms are incomplete, and FMF is therefore not diagnosed. Most misdiagnosed patients are operated on or biopsied. Before the use of colchicine, many patients developed renal dysfunction through amyloid accumulation. ${ }^{4}$ Amyloid fibrils of FMF (AA) are deposited in many organs, mainly in the kidney, leading to end stage renal failure. Continuous prophylactic colchicine treatment (1-2 mg/day) prevents febrile attacks in $90 \%$ of patients and amyloidosis in $2 / 3$ cases. $^{45}$ It can also reduce proteinuria and reverse the nephrotic syndrome and the amyloidosis. ${ }^{67}$ Misdiagnosis of FMF is thus linked with outbreaks of renal complications, which occur in $12-60 \%$ of untreated patients, so it is crucial to develop strategies permitting early diagnosis.

Recessive inheritance has been shown by many research teams and confirmed by us, ${ }^{8}{ }^{9}$ but the gene responsible for FMF (MEF), which has been mapped to chromosome $16 \mathrm{p} 13.3,{ }^{10}$ has not yet been isolated. Using linkage disequilibrium analysis, we recently narrowed the MEF region to $250 \mathrm{~kb}$ and discovered an ancestral haplotype in nonAshkenazi Jews. ${ }^{9}$ In the present report, we discuss the suitability of each MEF linked microsatellite marker (including four new ones) for routine genotypic diagnosis, and propose a quick and reliable tool for indirect FMF diagnosis/heterozygote testing. Two large families are presented to illustrate the reliability of the test.

\section{Materials and methods} PATIENTS

Blood samples were obtained from as many voluntary members as possible from each pedigree and a careful family history was drawn up. Individual members of all families were listed chronologically. This numbering scheme was adopted for the figures in the present paper for consistency with our previous haplotype 


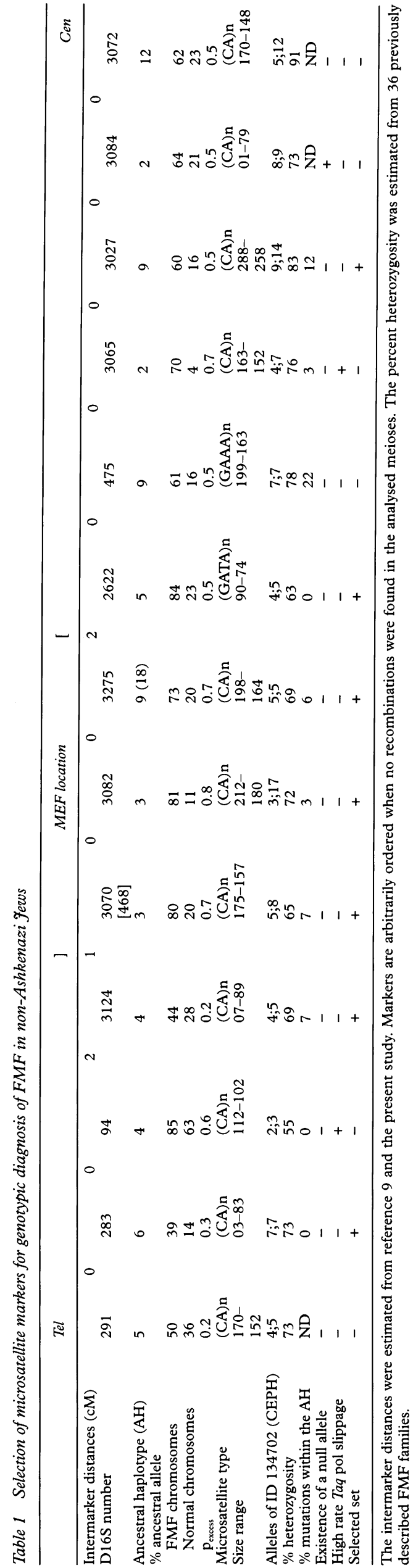

analysis. ${ }^{9}$ Marker characterisation and selection for genotypic diagnosis were investigated in a previously described 36 member nonAshkenazi Jewish family panel. ${ }^{9}$

The reliability of indirect genotypic diagnosis was evaluated in the Montpellier FMF family panel, including 26 families recruited in the initial study and seven additional North African Jewish families $(67,104,106,108,112$, 119 , and 122). A total of 308 people were genotyped in these 33 pedigrees.

DNA ANALYSIS

Genomic DNA was extracted from blood leucocytes according to established protocols. ${ }^{11}$ Genotyping was carried out using standard procedures. ${ }^{12}$ Information concerning primers was from the Genome Data Base (GDB).Subject 134702 from the CEPH (Centre d'Etude du Polymorphisme Humain) was genotyped in each set of reactions as an allele size reference.

\section{MARKER CHARACTERISATION AND STATISTICAL} ANALYSIS

Alleles were numbered according to their repeat content, with allele 1 representing the heaviest allele. The base pair sizes for the core 3-3-9 haplotype were $171 \mathrm{bp}, 208 \mathrm{bp}$, and 182 $\mathrm{bp}$, respectively. Allele frequencies and linkage disequilibrium $\left(p_{\text {excess }}\right)$ were estimated as described previously ${ }^{9}$ from the first family panel.

\section{Results}

SELECTION OF THE BEST MICROSATELLITE

MARKER SET FOR GENOTYPIC DIAGNOSIS

We used our previous data for fine MEF localisation $^{9}$ and also examined four microsatellite markers recently located in chromosome $16 \mathrm{p} 13.3$, that is, D16S134 and D16S3082, which cosegregated with D16S3070 in the CEPH family panel, and D16S468 and D16S2622 (GDB). It became clear that all markers were not of equal interest for genotypic diagnosis of the disease (table 1).

At the telomeric end of MEF, a cluster of three markers (D16S291, D16S283, and D16S94) was shown to be located quite far (3-4 cM) from the mutation. ${ }^{13}$ We selected D16S283, since D16S291 showed no alleles in significant linkage disequilibrium with the disease and D16S94 was not very informative. Although we found that marker D16S3124 had two alleles in linkage disequilibrium, it was still selected for diagnosis because no other marker was located within the 1-2 cM DNA segment telomeric to MEF.

We had already found two markers (D16S3070 and D16S3275) that never recombined with the gene throughout 310 meioses studied. As expected, we discovered no crossover between MEF and D16S3082. Its position relative to D16S3070 and D16S3275 therefore could not be determined on the genetic map. Allele 3 of D16S3082 displayed the strongest linkage disequilibrium $\left(\mathrm{p}_{\text {excess }}=0.8\right)$ with the disease (table 1). The core 3-3-9 (or 3-3-18) haplotype (D16S3070, D16S3082, and D16S3275, respectively) was present in $70 \%$ of mutated chromosomes, whereas it was never found in non-mutated chromosomes in our ini- 
tial non-Ashkenazi Jewish population (not shown). We discovered that D16S468 contained the same microsatellite sequence as D16S3070, and that D16S134 was centromeric to D16S3072 in our meioses (not shown). D16S468 and D16S134 were therefore abandoned.

At the centromeric end of MEF and cosegregating with D16S475, we found that D16S2622 displayed one allele in excess in FMF haplotypes (allele 5), as we previously showed for D16S475, D16S3065, D16S3027, D16S3084, and D16S3072 (table 1). Three of these centromeric markers were not considered suitable for routine use. Marker D16S3065, which exhibited a high rate of Taq polymerase slippage artefacts, was sometimes difficult to interpret. Marker D16S3084 showed a null allele in 3\% of non-Ashkenazi-Jewish chromosomes. Since none of the bracketing loci was found to be deleted (not shown), the null allele probably reflected either a small deletion at this locus or the presence of a polymorphic sequence in one of the primers used for marker D16S3084 PCR amplification. D16S475, which bears the highest number of alleles (size range 199-163), also displayed the highest mutation rate. Our current selected set for FMF heterozygote analysis and diagnosis thus includes two markers telomeric to $\mathrm{MEF}$ (D16S283 and D16S3124), three markers showing no recombination with the disease (D16S3070, D16S3082, and D16S3275), and two markers centromeric to the gene (D16S2622 and D16S3027). Moreover, a whole family could be rapidly explored on a single gel by loading two or three different sized PCR fragments (table 1) in the same track.

FAMILY REPORTS AND HAPLOTYPE ANALYSIS Genotyping offers new potential for biological exploration of FMF in non-Ashkenazi Jewish populations, as illustrated by the present results on the large and well documented families 21 and 75 . They had both emigrated from North Africa to France in the early 1960s.

Family 75 originated from Bizerte (Tunisia) and included eight inbreeding loops. When this family was recruited, FMF was diagnosed in $18 / 52$ living members as shown in fig 1 . Most patients experienced the disease in early childhood, except subjects (ID Nos) 1, 22, and 82 who began suffering from FMF between the ages of 30 and 40 . Since the disease often mimics surgical emergency, subjects 4 and 32 underwent appendectomy and cholecystectomy, respectively, before FMF was diagnosed. All subjects, except No 78, displayed the full clinical symptomatology of FMF and responded well to continuous colchicine treatment. In addition, subject 13 presented secondary renal amyloidosis and underwent haemodialysis. FMF was diagnosed in subject 78 on the following criterion: at the age of 55 , he experienced abdominal and feverish attacks which lasted four to five years, then the episodes resolved spontaneously. No laboratory tests were done and there were no other attempts to diagnose his disorder.
All members of one branch of the family were affected: the two parents (subjects 25 and 82) linked by two inbreeding loops and their three children (subjects 83, 84, and 85). Examination of their haplotypes showed that subject 25 had inherited two identical mutated chromosomes, and one copy was also transmitted to his wife, subject 82 (fig 1). This haplotype was again duplicated in two of their children (Nos 83 and 85). The FMF mutation was easily traced in this pedigree, even when restricting the analysis to the panel of seven markers described above. Subject 78, who presented "late onset FMF" had only one known FMF chromosome. Since three of his sibs (subjects 77,79 , and 81 ) were haploidentical without any FMF symptoms after the age of 50 years, the present results strongly suggest that subject 78 was precipitately diagnosed, owing to the high number of FMF cases in this family.

All FMF bearing chromosomes in family 75 were of ancestral origin, at least at the three core loci (D16S3070, D16S3082, D16S3275), except for one carried by subject 9 and her daughter No 16 (alleles 5-11-5 respectively at the three core markers). Subject 5 , who was symptom free, showed the same genotype as her affected sister (No 4). She is probably concerned by the known low, sometimes age dependent, penetrance of the disease in affected females, ${ }^{14}$ since she was 25 when she was genotyped, and her affected mother (No 1) and sister (No 22) only showed symptoms of the disease after the age of 30 years.

Family 21 (fig 2) illustrates most situations that can be encountered in genetic counselling. Almost all members of this family were of Algerian origin. Subject 31 had the full range of FMF symptoms (fever, abdominal and joint pain) at the age of 6 years. Although subjects 11 and 12 also displayed typical FMF, the older sister (No 11) had had an appendectomy before FMF was diagnosed, because of acute abdominal attacks during adolescence. Her major symptoms then switched to episodes of shortness of breath, probably owing to pleuritis. Subject 28 endured the most severe form of FMF in the pedigree (fever reaching $41^{\circ} \mathrm{C}$, extreme asthenia, peritonitis, pleuritis, and orchitis). He had the first attacks at the age of 10 months and also underwent unnecessary surgery. The diagnosis was delayed until the age of 3 years. Subject 29 suffered from recurrent episodes of joint pain associated with fever $\left(38-39^{\circ} \mathrm{C}\right)$ and had sterile meningitis at the age of 4 years. FMF was immediately diagnosed in view of the family history. The younger brother (No 30) showed transient episodes of abdominal and thoracic discomfort in early childhood. It was also immediately assumed that he suffered from FMF. All six patients were treated with colchicine on diagnosis. Subject 17 experienced bearable recurrent abdominal pain and six episodes of unexplained fever during adolescence. FMF was a logical diagnosis since her paternal (No 31) and maternal (Nos 11 and 12) cousins suffered from this disorder. She is now 37 and no longer has symptoms, 

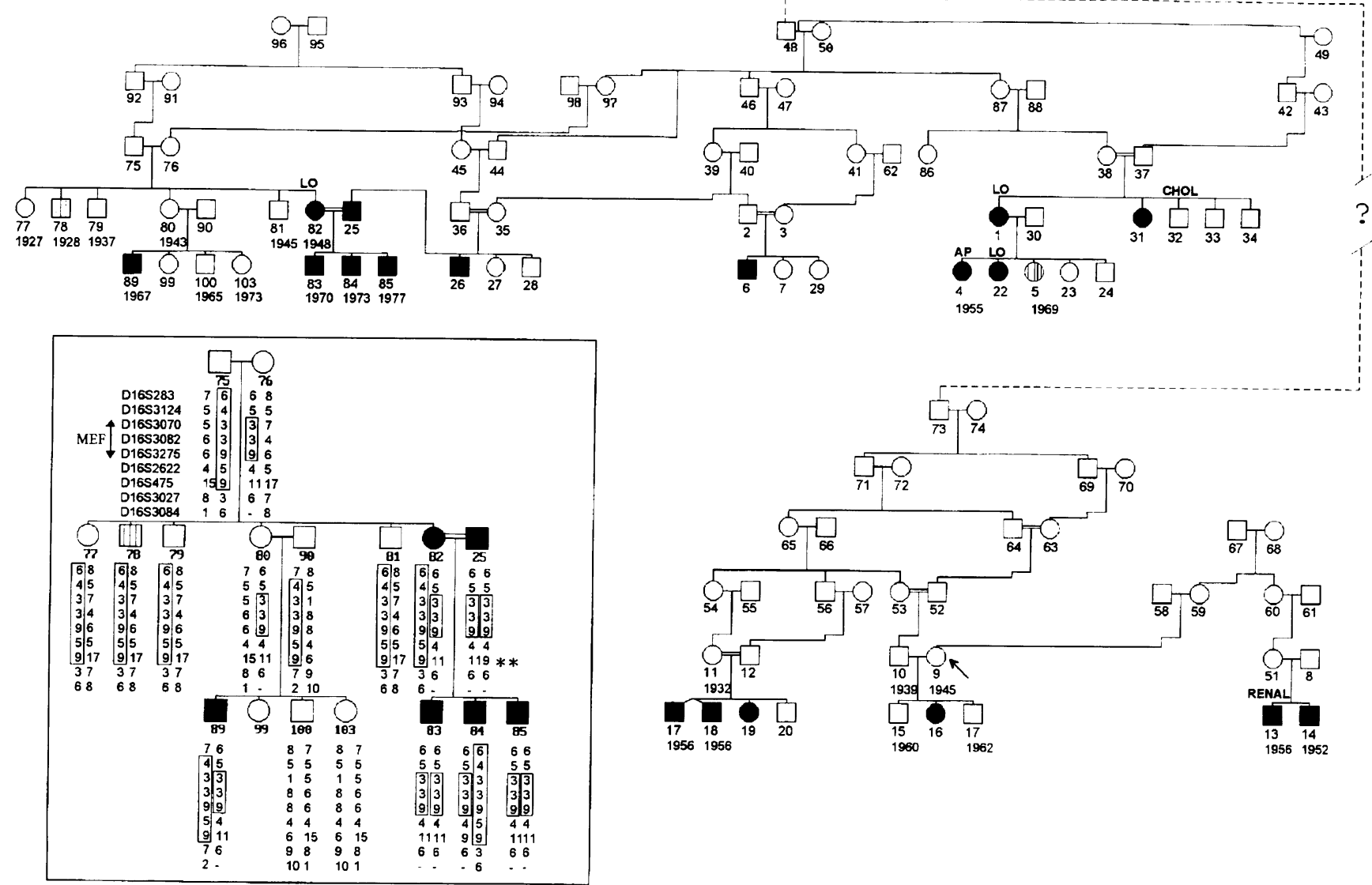

Figure 1 Family 75: a pedigree with eight inbreeding loops. The exact relationship between the upper (subject 48) and the lower (subject 73) part of the pedigree is currently unknown (?). Subject 48 married twice (to subject 50, then to 49). Patients'years of birth are indicated beneath their individual numbers when available. Particular clinical traits are specified above the individual numbers: LO: late onset, CHOL: cholecystectomy, AP: appendicectomy, RENAL: renal complications. Filled symbols indicate phenotypically diagnosed living members. Hatched symbols correspond to patients (5 and 78 ) with apparent genotype/phenotype inconsistency (see text). All FMF chromosomes were of the ancestral type, except in subject 9 (arrowed). Haplotypes of the most inbred branch of this family are detailed in the bottom left corner. The ancestral part of each haplotype is boxed. Marker D16S475 recently mutated in family 75 as shown by the heterozygous status of subject 25 at this locus $(* *)$. A null allele (-) was present at marker D16S3084.

although she never took colchicine. None of her sibs (age range 30-36) had symptoms of FMF.

The genotyping results are shown in fig 2 . A number of different FMF haplotypes were found in this pedigree. Subjects 17 and 30, who showed discrete symptomatology, carried only one known FMF chromosome. We found that No 17 had inherited the maternal FMF chromosome but neither of the two FMF chromosomes carried by her paternal cousin No 31, which were found to be of unrelated origin. Based on this result, along with the haploidentity found in her symptom free sister No 19, there was little chance that her past health problems were related to FMF, although this could not be definitely rejected. Genotypes found in subjects 28 and 30 are consistent with a correct diagnosis in the former (two ancestral chromosomes) and a misdiagnosis in the latter (no FMF symptoms in his mother, No 24, or aunt, No 23, who had the same haplotypes).

Unlike in family 75 , only one patient (No 28 ) among those clinically diagnosed in the initial study exhibited two Jewish FMF ancestral haplotypes. At this time, only three independent FMF foci (Nos 31, 11, and 12, Nos 28, 29, and 30) were known, and the father, sister, and brothers of No 27 had not yet been genotyped. Interestingly, an ancestral haplotype was discovered in subjects 20 and 27, which was not inherited from a known heterozygote. Further detailed interrogation disclosed that No 20's grandmother's first cousin died of FMF linked renal amyloidosis and that the sister of No 27 (No 71) had recently complained of abdominal pain recurring every morning (without any other symptoms). Since the diagnosis of FMF was suspected in No 71, we genotyped her father and the missing sibs and showed that she carried neither the maternal known FMF mutated chromosome nor the ancestral paternal haplotype, thus prompting further laboratory testing and avoiding precipitate colchicine treatment. Lastly, the heterozygous status of a couple (Nos 14 and 25) was established, therefore justifying close follow up of their future children.

RELIABILITY OF THE INDIRECT GENOTYPIC DIAGNOSIS IN NON-ASHKENAZI JEWISH FAMILIES To investigate whether the use of the set of seven highly polymorphic markers could be generalised, we examined two possible strategies for indirect genotypic diagnosis of FMF as a function of the number of ancestral haplotypes in the FMF affected proband (table 2).

As for any recessive disease for which the causative gene is still unknown, diagnosis may be done by classical linkage analysis, which requires available mutated DNA of paternal and maternal origin. To evaluate the accuracy 


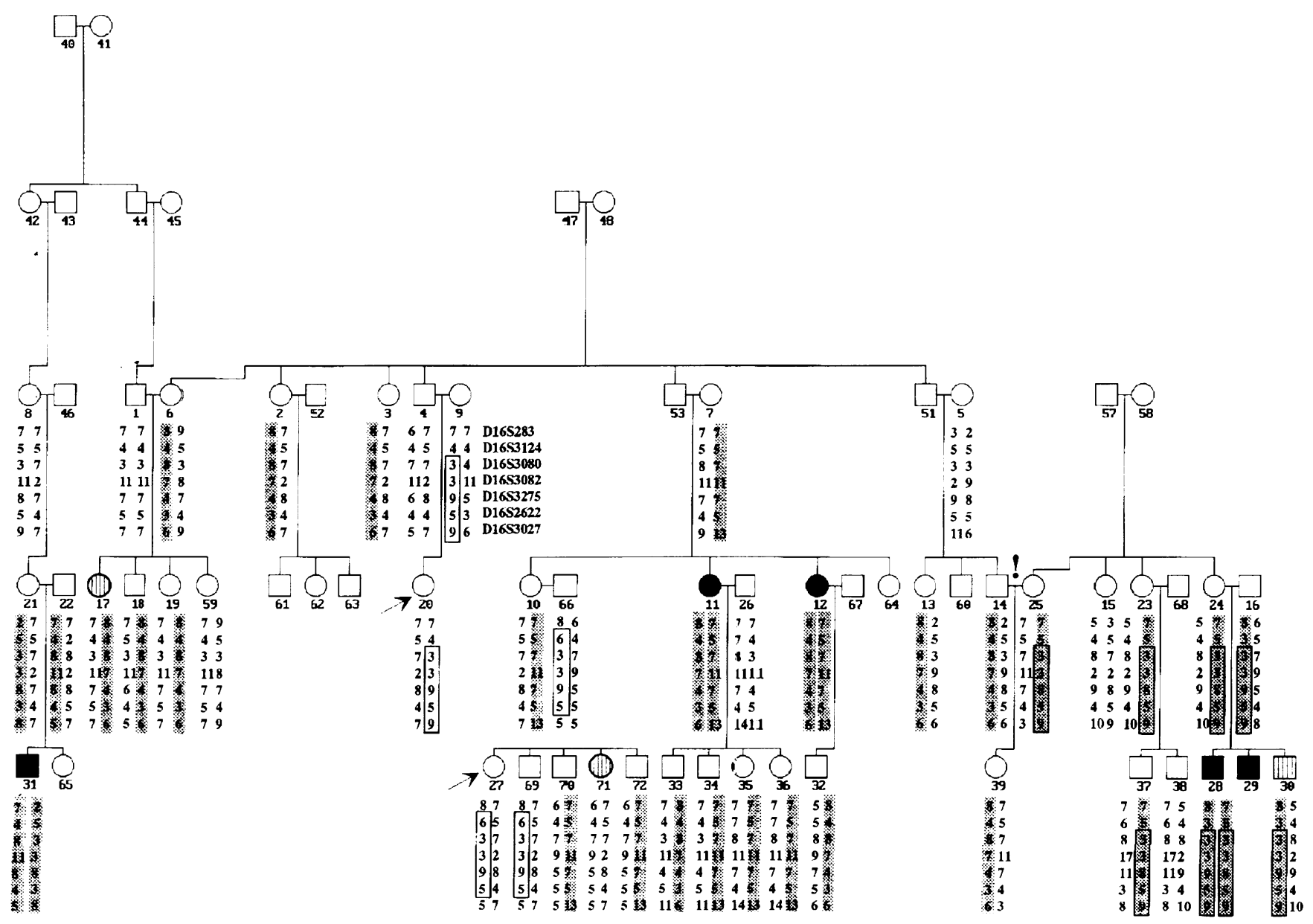

Figure 2 Family 21: a pedigree with most indications for genotypic diagnosis. Symbols are as in fig 1. FMF mutated chromosomes are shown in grey. Allele 8 at locus 3275 in subject 28 was most probably derived from a mutation since the ancestral haplotype was preserved down to locus D16S3072. People who were found to carry one ancestral haplotype are arrowed. A heterozygous couple was discovered.

of such diagnosis for FMF, we examined the informativeness of the seven markers in the clinically diagnosed patients. Linkage analysis was informative in more than half of the patients (53\%) when we analysed the three core microsatellites (D16S3070, D16S3082, and D16S3275) alone, in $97 \%$ of patients when adding the two bracketing markers D16S3124 and D16S2622 at 1-2 cM from $\mathrm{MEF}$, and in $100 \%$ of cases with the combined use of our set of seven markers. The results obtained with three informative markers at 250 $\mathrm{kb}(<1 \mathrm{cM})$ from the causative mutation, provide a recombination risk of less than $1 \%$.

The increasing number of sporadic cases owing to smaller family size prompted us to assess the impact of the founder effect in FMF patient genotypes (table 2). Indeed, $63 \%$ of Jewish patients of North African origin had both chromosomes of the ancestral type (3-3-9 and $3-3-9$ or 3-3-9 and 3-3-18) and this rate reached $73 \%$ when one mutation was allowed at the three core loci, provided that our two proposed bracketing markers also exhibed ancestral alleles in linkage disequilibrium. Twenty-two percent of patients carried one ancestral haplotype, and as few as $5 \%$ had no ancestral mutated chromosome. Even more specific, allele 18 of D16S3275 was only noted in FMF mutated chromosomes ${ }^{9}$ (present study). Among the 101 independent normal chromosomes studied in our latter family panel, only one apparently non-mutated chromosome exhibited the 3-3-9 haplotype (subject 1 , family 14). Thus, diagnosis based on detection of the homozygous status for the 3-3-9 haplotype in the non-Ashkenazi Jewish patients resulted in a very low risk of error $\left(2.10^{-5}\right)$, the chance occurrence of each ancestral allele being $0.2,0.11$, and 0.2 at $\mathrm{D} 16 \mathrm{~S} 3070$, D16S3082, and D16S3275, respectively, in this population (table 1 ).

We then retrospectively compared the potential benefits of each linkage approach in our FMF affected population. The initial clinical diagnoses per patient were plotted against the corresponding genotypic statuses established in the present study (table 2). From a total of 84 clinically diagnosed patients who could be genotyped, 76 (90\%) exhibited an informative genotype with either or both linkage approaches. Genotypes were found to be consistent with clinical diagnosis in all patients except subject 78 , family 75 and subject 30 , family 21 , who exhibited one mutated ancestral chromosome and one apparently non-pathogenic chromosome. Since the three markers that never recombine with the disease gene were informative in these two patients, FMF may be genotypically refuted with less than $1 \%$ risk, which a postiori might explain the misdiagnosis described earlier. It is noteworthy that the disequilibrium linkage approach alone was 
Table 2 Clinical diagnosis and genotypic status in 33 non-Ashkenazi fewish pedigrees

\begin{tabular}{|c|c|c|c|c|c|c|}
\hline \multirow{2}{*}{\multicolumn{2}{|c|}{$\begin{array}{l}\text { Known mutated } \\
\text { chromosome (s) }\end{array}$}} & \multicolumn{5}{|c|}{ Clinical FMF } \\
\hline & & \multicolumn{3}{|c|}{ Diagnosed $(n=84)$} & \multirow[b]{2}{*}{ Suspected $(n=8)$} & \multirow[b]{2}{*}{ No symptoms $(n=4)$} \\
\hline Non- $A H$ & $A H$ & $1 \mathrm{sib}$ & 2 sibs & 3 sibs & & \\
\hline & & & $6(1,2)$ & & & \\
\hline & & $21(28)$ & $20(4,6)$ & & & \\
\hline & & $23(1)$ & $22(2,5)$ & & & \\
\hline & & $\overline{55(5)}$ & $55(11,12)$ & & & \\
\hline & & $57(2)$ & $57(4,5)$ & & & \\
\hline & & $65^{\star}(34)$ & & & & \\
\hline & & $65(43)$ & & & & \\
\hline & & $\underline{69(6)}$ & $60(1,4)$ & $60^{\star}(8,9,11)$ & & $60^{\star}(12)$ \\
\hline & & $69(8)$ & $65(25,27)$ & $61(1,2,3)$ & & \\
\hline & & $71(5)$ & $75^{\star}(1,31)$ & $75^{\star}(83,84,85)$ & & \\
\hline & & $71(10)$ & & & & \\
\hline & 2 & $75(4)$ & & & & $75(5)$ \\
\hline & $73 \%$ & $75(6)$ & $75(13,14)$ & & & \\
\hline & & $75^{\star}(25)$ & $75^{\star}(18,19)$ & & & \\
\hline & & $75(82)$ & & & & \\
\hline & & $75(89)$ & & $85(3,5,7)$ & & \\
\hline & & $78(2)$ & $81(2,3)$ & & & \\
\hline & & $78(7)$ & & & & \\
\hline & & $93(15)$ & & $93(3,5,7)$ & & \\
\hline & & $94(3)$ & & & & \\
\hline & & $\underline{105(5)}$ & & & $\underline{122(7)}$ & \\
\hline & & $106(3)$ & & & & \\
\hline & & $\overline{108(1)}$ & & & & \\
\hline & & $119(10)$ & & & & \\
\hline & & $108(4)$ & & & & \\
\hline & & $119(3)$ & & & & \\
\hline \multirow[t]{10}{*}{1} & 1 & $61(16)$ & $61(7,8)$ & & & \\
\hline & & $61(20)$ & $62(4,5)$ & & & \\
\hline & & $61(26)$ & & & $61(21,25)$ & \\
\hline & & $\underline{64(3)}$ & $83(2,3)$ & & & $83(1)$ \\
\hline & & $67(3)$ & & & & \\
\hline & & $68(3)$ & & & & \\
\hline & & $73(5)$ & & & & \\
\hline & & $75(16)$ & & & & \\
\hline & & $91(3)$ & & & $91(4)$ & \\
\hline & & $\underline{104(1)}$ & & & & $14(1)$ \\
\hline 2 & & $\underline{14(2)}$ & & & & \\
\hline \multirow[t]{3}{*}{$5 \%$} & & $21(31)$ & $21(11,12)$ & & & \\
\hline & 1 & $21(30)$ & & & & \\
\hline & & $75(78)$ & & & $94(4)$ & \\
\hline \multirow[t]{2}{*}{1} & & & & & $21(17)$ & \\
\hline & $1+?$ & & & & $112(1)$ & \\
\hline 0 & 0 & & & & $\underline{21(71)}$ & \\
\hline
\end{tabular}

The patients are depicted as follows: family number (sib numbers). A number of families had multiple FMF foci: $21,55,57,60,61$, $65,69,71,73,75,78,93,108,119$. Isolated cases are underlined. Consanguineous patients are indicated by an asterisk. Subject 1 (family 112) had one ancestral haplotype and one ancestral recombination within the core markers $(1+$ ?).

informative for $6 / 12$ isolated cases present in this panel.

Eight additional patients were clinically suspected and asked to undergo genotypic diagnosis. The benefit of the genetic test was clear for seven of them. One isolated case (subject 7 , family 122) was diagnosed with a risk of $<2.10^{-5}$ by disequilibrium linkage analysis alone, three were confirmed (Nos 21 and 25, family 61 and No 4, family 91), and two patients were found to be at low risk (No 71, family 21 and No 4, family 94 ) by both standard and disequilibrium linkage analysis. Subject 17 (family 21) could not be assessed through disequilibrium linkage analysis and was investigated using standard linkage analysis alone (see above). Subject 1 (family 112) could not be confirmed because she had an ancestral recombination within the core markers.

Finally, only $2 \%(4 / 216)$ of genotyped FMF symptom free people were found to carry two mutated or ancestral chromosomes. In Nos 12 (family 60), 5 (family 75), and 1 (family 83), random coincidental allelic distribution was unlikely since, in each person, the two haplotypes were identical to their affected sister all along the seven markers. Subjects 5 (family 75 ) and 12 (family 60) were younger than 30 and might have late onset of the disease, and Nos 1 (families 83 and 14) were older than 45 and thus age independent penetrance was probably involved.

\section{Discussion}

To date, 16 highly polymorphic markers have been shown to span the 5-6 cM D16S94D16S523 MEF region. Three of the four newly analysed $16 \mathrm{p} 13.3$ microsatellite markers (D16S468, D16S3082, and D16S2622) were inserted into the group closest to MEF. Of particular interest, D16S3082, which never recombined with the gene, joined the core markers (D16S3070-D16S3275). Moreover, allele 3 of D16S3082 exhibited the highest linkage disequilibrium with MEF (table 1), indicating that it is the best candidate region for gene search and clinical use. We compared the properties of each marker and selected seven of them for FMF testing in non-Ashkenazi Jews. Of course, direct identification of MEF muta- 
tions remains essential for certain diagnosis. However, this potentially fatal disease can be efficiently treated, thus justifying the development of biological analyses that are more specific than current evaluation of inflammation (increased sedimentation rate, etc).

Using classical linkage analysis with the proposed highly informative set of markers, the parental origin of the two chromosomes could be confirmed in $100 \%$ of cases in the first family panel and in $99 \%$ of cases in the second (in subject 71 , family 21 , one more telomeric marker was needed). This approach is restricted to cases where DNA from FMF affected relatives is available from both parental sides. However, we obtained more accurate indirect diagnoses through the strong founder effect noted in non-Ashkenazi Jewish families. With $73 \%$ of FMF patients exhibiting homozygosity for the ancestral haplotype in this population, the risk of error was much lower with the linkage disequilibrium approach, which is especially appropriate for isolated cases. In practice, we propose the following two step strategy for genotypic FMF tests: (1) search for the ancestral haplotype in the putative FMF chromosomes, then (2) familial studies if fewer than two ancestral haplotypes are found. The clear limit of the disequilibrium linkage approach is that, in an isolated person in whom FMF is suspected, the lack of 3-3-9 homozygosity cannot refute the diagnosis.

Besides the advantages of genotyping for FMF diagnosis in people with symptoms, the present report shows that the proposed test will also be of use for care management. Screening heterozygous couples in families at risk, such as subjects 14 and 25 in family 21 , will allow early detection of children who have inherited two mutated alleles. Presymptomatic diagnosis can now be expected, since we could predict the heterozygous status of subject 9 (family 21) before knowing that she had one affected relative, although the origin of her ancestral haplotype has not yet been definitely proven, as no DNA is available from her dead cousin. We were also able, through detection of ancestral core alleles, successfully to confirm the diagnosis for an isolated case (No 7, family 122). Following genotypic diagnosis, this patient started colchicine treatment, which immediately stopped her febrile and painful attacks. Two ancestral haplotypes were discovered in two young FMF symptom free women (No 12, family 60 and No 5, family 75). Clinical follow up and regular laboratory test controls of renal function will enable early detection of possible form II FMF, which is characterised by inaugural renal amyloidosis.

Apart from the clinical interest, fundamental information, such as the number of genes involved in the disease, will be extracted through general application of genetic tests in the population concerned. Symptoms consistent with FMF were detected in three patients carrying only one mutated chromosome (No 30 , family 21 , No 78 , family 75 , and No 4 , family 94). Although dominant inheritance of FMF was mentioned recently, ${ }^{15}$ the trait was clearly recessively transmitted in all our pedi- grees. However, mild expressivity of the disease in heterozygotes remains possible. Family 75 showed extreme FMF forms (with full or no symptomatology, with or without renal complications) in patients carrying identical genotypes (two ancestral haplotypes). Locus homogeneity, despite the existence of various clinical forms of the disease, has already been reported using linkage with the $\alpha$ globin complex. ${ }^{8}$ The present data suggest, in addition, that identical mutations are involved in the onset of FMF in this family, implying that FMF phenotypic heterogeneity is the result of the interaction of MEF with still unknown modifier genes or environmental factors or both. Once the gene has been identified, it will be interesting to list the mutations and correlate them with clinical FMF patterns.

Burdensome surgical diagnosis, delayed treatment (exposing patients to renal failure), or, conversely, excess diagnosis (leading to unnecessary life long treatment) could be avoided. The reliable genotypic diagnostic test that is now available should be proposed to all families at risk for or with clinical symptoms consistent with FMF, with the linkage analysis strategy adapted to each specific case. Until direct genotypic analysis is available, we plan to extend the present indirect test to other ethnic groups affected by the disease.

We are indebted to the families and physicians without whom he study would not have been possible. We are grateful to Proessor Lambert and to Dr Vallet for reviewing the manuscript. This work was supported by the Association Française pour la Myopathie, the Association pour le Développement de 'Evaluation Thérapeutique, and the Centre Hospitalier de Montpellier.

1 Heller H, Sohar E, Sherf L. Familial Mediterranean fever (FMF). Arch Intern Med 1958;102:50-71.

2 Daniels M, Shohat T, Brenner-Ulman A, Shohat M. Familial Mediterranean fever: high gene frequency among the non-Ashkenazic and Ashkenazic Jewish populations in Israel. Am 7 Med Genet 1995;55:31 1-14.

3 Sohar E, Gafni J, Pras M, Heller H. Familial Mediterranean fover: a survey of 470 cases and review of the literature. $A m$ fever: a survey of 470 case

4 Zemer D, Pras M, Sohar E, et al. Colchicine in the prevention and treatment of the amyloidosis of familial Mediterranean fever. $N$ Engl f Med 1986;314:1001-5.

5 Livneh A, Zemer D, Langeitz P, et al. Colchicine treatmen of AA amyloidosis of familial Mediterranean fever. Analysis of factors affecting outcome. Arthritis Rheum 1994;37 1804-11.

6 Hojberg AS, Mertz H. Nephrotic syndrome in familial Mediterranean fever: effect of colchicine therapy. Ugeskr Laeger 1995;157:4035-7.

7 Rozenbaum M, Rosner I. Regression of amyloidosis with colchicine in familial Mediterranean fever in an Ashkenazi patient. Clin Exp Rheumatol 1995;13:126.

8 Shohat M, Bu X, Shohat T, et al. The gene for familial Mediterranean fever in both Armenians and nonAshkenazi Jews is linked to the alpha globin complex on Ashkenazi Jews is linked to the alpha globin complex on 992:51:1349-54

9 The FMF French consortium. Localization of the familial Mediterranean fever (FMF) gene to a $250 \mathrm{~kb}$ interval in non-Ashkenazi Jewish haplotypes. Am $f$ Hum Genet 1996;59:603-12.

10 Pras E, Aksentijevich I, Gruberg L, et al. Mapping of a gene causing familial Mediterranean fever to the short arm of chromosome 16. N Engl f Med 1992;326:1509-13.

11 Sambrook J, Fritsch EF, Maniatis J. Molecular cloning: a laboratory manual. $2 \mathrm{~d}$ ed. Cold Spring Harbor, NY: Cold Spring Harbor Laboratory Press, 1989.

12 Gyapay G, Morissette J, Vignal A, et al. The 1993-94 Généthon human genetic linkage map. Nat Genet 1994;7:246339.

13 Aksentijevich I, Pras E, Gruberg L, et al. Refined mapping of the gene causing familial Mediterranean fever by linkage and the gene causing familial Mediterranean fever by linkage and

14 Rogers D, Shohat M, Petersen G, et al. Familial Mediterranean fever in Armenians: autosomal recessive inheritance with high gene frequency. Am $\mathcal{F}$ Med Genet 1989;34:168-72.

15 Yuval Y, Hemo-Zisser M, Zemer D, et al. Dominant inheritance in two families with familial Mediterranean fever (FMF). Am f Med Genet 1995;57:455-7. 